\title{
Quality of life and self-stigma of schizophrenia patient's caregiver tool: Development and validation using classical test theory and Rasch analysis
}

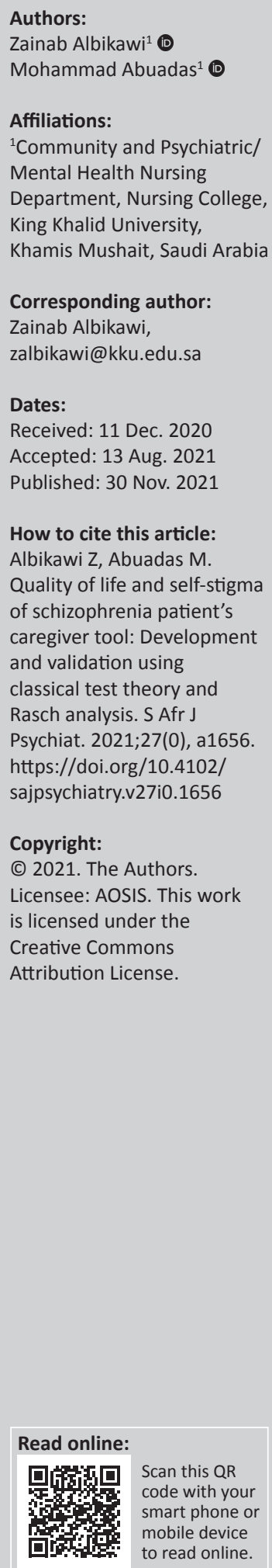

Background: Providing care for schizophrenia patients is complex, and it requires dealing with various psychosocial burdens.

Aim: To develop and validate a tool that measures the quality of life and self-stigma (SS) of the schizophrenia patient's caregiver (QLSSoSPC).

Setting: Outpatient psychiatric services clinics in Saudi Arabia.

Methods: The current study used a methodological cross-sectional design. A sample of 205 schizophrenia patients' caregivers was recruited by using a convenient sampling method. Classical Test Theory and Rasch Analysis approaches were used.

Results: The developed tool has proven acceptable level of reliability and validity. The analysis confirmed seven-factor structure accounted for $74.4 \%$ of the total variance. Cronbach's reliability statistics for the developed tool were satisfactory and ranged from 0.80 to 0.91 .

Conclusion: The psychometric properties of the QLSSoSPC tool supported its prospective use and allowing us to recommend the implementation of the tool on behalf of clinical and research purposes.

Keywords: schizophrenia; caregivers; validity; reliability; Rash analysis; quality of life; self-stigma; psychological well-being.

\section{Introduction}

Schizophrenia is a complex and multi-causal syndrome. Its prevalence is about 1.5 per 10000 people. $^{1}$ Age of onset is usually during adolescence; childhood and late-life onset is rare. Slightly more males are diagnosed with schizophrenia than females $(1.4: 1){ }^{2}$ and females tend to be diagnosed later in life than males.

Schizophrenia is considered a serious psychological disorder that influences the lives of patients and patients' caregivers. Caregivers' responses regarding having a family member with schizophrenia disorder are care burden, shame about illness, uncertainty about the disease and stigma. ${ }^{3}$ The responsibility of caregivers has gained a major function, as a consequence of increasing shift of psychiatric healthcare facilities to the community. Psychiatric patients received moderately short inpatient healthcare and early discharge from the healthcare institution adds to the important role of caregivers. ${ }^{4}$

A caregiver is defined as a family individual who is existing for more than 1 year with the patient and has directly participated in patient's daily living activities, social interaction and psychiatric healthcare issues. ${ }^{5}$ Schizophrenia also influences the performance and health of family caregivers, mostly because they have assumed the responsibilities that were performed previously via psychiatric healthcare institutions. ${ }^{6}$

The life-quality of family caregivers is greatly affected by the care provided to the schizophrenia patient. ${ }^{7}$ Schizophrenia patient's caregivers have been observed to have low quality of life, particularly when experiencing a significant burden. ${ }^{8}$ In addition, caregivers' depressing experience may influence their capability to take care of their patients. ${ }^{9,10}$ Recent evidence emphasised that improving quality of life has significant impact on the health of both the caregivers and their patients'. In spite of the wealth of literature that addressed specific issues related to care giving, little has been conducted to explore quality of life amongst schizophrenia patient's caregivers. Moreover, 
there is few studies that developed a tool to measures the life-quality of the schizophrenia patient's caregiver. ${ }^{78}$

Providing care for schizophrenia patients is complex and technical, and it requires dealing with various psychosocial burdens. Family members often assume the care giving role with little or no preparation and without considering whether they have enough psychological and social preparations. ${ }^{11,12}$. Stigma is one of the main demanding psychosocial burdens experienced by the caregivers of the mentally ill patients. ${ }^{13,14,15,16}$ A large study of family caregivers in Morocco found that caregivers experienced a high level of perceived stigma. ${ }^{17}$

Consequently, care-giving (i.e. providing care to patients with schizophrenia) has a substantial impact on family caregivers' social and mental well-being, and can negatively influence patient and caregiver health outcomes. ${ }^{14}$ Other researchers found a significant association amongst caregivers' self-stigma, psychological stress and quality of life. ${ }^{18,19,20}$ A recent study found a negative significant relationship between self-stigma and quality of life, where those who had higher self-stigma were more expected to have poorer quality of life. ${ }^{21}$

There is a strong consensus supporting that caring for an individual with schizophrenia is burdensome and stressful to family caregivers and contributes to significant reduction in quality of life for family members. Studies agreed with the consideration that quality of life, especially of family caregivers of schizophrenia patients, is mainly linked to many essential factors, that include: (1) individual wellbeing, (2) psychological status, (3) functional abilities, (4) social interaction, (5) occupational status, (6) financial status and (7) physical health (PH). ${ }^{22}$ As these studies indicate, it is essential to have well-prepared caregivers and to promote their quality of life because caregivers play a critical role in helping patients deal with the effects of mental illness and its treatment or maintain their health.

To provide an understanding of the quality of life for family caregivers of patients with schizophrenia and their need for better preparation and psychosocial care, this state-of-thescience examines the most essential domains of quality of life for family caregivers. Therefore, there is a need to develop and validate a tool for evaluating quality of life and self-stigma (SS) for the schizophrenia patient's caregiver. Up to now, reliable and valid tool that clearly and sufficiently incorporates the concept of SS; besides the main domains of quality of life have not been available, that's why the quality of life and SS of the schizophrenia patient's caregiver (QLSSoSPC) represent a significant new development. The current study aimed to develop and validate a tool that measures the QLSSoSPC.

\section{Methods}

\section{Study design}

The current study used a methodological cross-sectional design. $^{23}$

\section{Sample and data collection}

Sample size was considered in line with the recommendations of Nunnally ${ }^{24}$ who advised a graded scale to determine sufficient sample size for tool development as the following: poor $=100$, fair $=200$, good $=300$. Out of 310 participants who were selected by convenience sampling methods and were asked to join the study, a sample of only 205 participants who matched the study inclusion criteria participated in the existing study. The inclusion criteria were: (1) being the father, mother, sisters or brothers of patients with schizophrenia, (2) schizophrenia patients being diagnosed based on Diagnostic and Statistical Manual of Mental Disorders (DSM-IV) criteria, (3) caregivers' age of 18 years old or more and (4) being able to speak and read Arabic language.

Data were collected and the participants were recruited from two psychiatric outpatient's clinics in Saudi Arabia. Participants were approached and screened for eligibility by: (1) accessing their medical health files, (2) identification of first-degree relative caregivers who take care of schizophrenia patients. The researchers invited and distributed the questionnaires to the eligible caregivers. Consent forms and cover letters were enclosed with the questionnaires. The researcher included full details of the study, information that participation was anonymous and voluntary and contact information for the researchers. The current study was approved by the Research Ethics Committee of the institution.

\section{Measures}

The study data were collected through the use of the following tools. The first tool was used to collect the socio-demographic characteristics of the caregivers (gender, age, number of family members and occupation). The second tool was used to collect the clinical information about schizophrenia patients (type of schizophrenia, age at diagnosis, duration of schizophrenia). The third tool was the Arabic version of the short-form of the World Health Organization's quality of life (WHOQOL-BREF 1998). ${ }^{25}$ The WHOQOL-BREF contains 26 -items that cover four domains: physical Health (PH, 7 items), psychological (Ps, 6 items), social relationships (SR, 3 items) and environment (E, 8 items). As no cut-points exist to categorise quality of life measured by WHOQOL-BREF, the final score ranges from 0 to 100 . The fourth one is a researcher-developed QLSSoSPC.

\section{Tool development procedure}

The final draft of the QLSSoSPC was developed in two stages: (1) qualitative stage in which items were generated, and (2) quantitative stage in which items were reduced and the QLSSoSPC was validated. ${ }^{26}$ The tool was originally developed in Arabic language since all participants were from Saudi Arabia. The study participants were different in the two stages. 


\section{Qualitative stage}

A focus group design was used to investigate the family caregivers' view and experiences about their quality of life when caring of schizophrenia patients. A focus group method is particularly suitable for obtaining information from several perspectives. ${ }^{23}$ According to Morgado and Meireles, ${ }^{27}$ the qualitative information, derived from direct participants' observations such as participants' interviews, can be used to recognise the domain items.

The participants from which the sample was drawn consisted of 15 caregivers who were arranged in three groups. Initially, the authors primarily employed information from the literature review and pre-existing scales to generate discussion questions that reflect the main domains related to the quality of life and self-stigma. The topics developed included 12 open-ended questions that were related to main domains. These questions were used to stimulate discussion. Those focus group discussions focused on quality of life and self-stigma as perceived by family caregivers who are caring of patients with schizophrenia. The authors used those discussions to establish the wording in question stems and the range of answer choices. All focus group discussions were recorded and thoroughly transcribed. The qualitative content analysis led to the emergence of 57-items from the focus group data.

After formulating the initial draft of items, the 15-caregivers were asked to give comments on any part of the tool such as: response options, content and tool items wording that they felt was unrelated or needs any improvement. Study items that were ambiguous, misunderstood were withdrawn or reworded that resulted in development of a preliminary tool that comprised 51 items. Finally, the focus group discussions guaranteed that the tool is the reflection of their experiences as the schizophrenia patient's caregiver.

\section{Quantitative stage}

Content validity: A panel of experts was invited to be involved in the content validity assessment. Six experts in psychiatric and mental health field evaluated the content validity of the first version of QLSSoSPC tool in terms of its relevance, clarity, meaningfulness and completeness using a two-step review process. In the first step, the list of all items was given to the experts, to evaluate and modify these potential items based on the following two criteria: (1) were the items suitable and consistent with the domain? and (2) was the phrasing of the items concise and accurate?

According to the suggestions made by the experts, the first version of the tool was reduced to 30 -items. In the second step, the revised draft of questionnaire was judged for relevance using a 4-point ordinal scale (1 - not relevant, 2 - somewhat relevant, 3 - quite relevant, 4 - highly relevant). The panel of experts was selected carefully and had the following credentials: (1) two experts were full professors of psychiatric and mental health nursing, two were clinician experts in psychiatric and mental health and the others were assistant professors of community health nursing; and (2) all experts had numerous publications related to psychiatric, mental health and community care. The following criteria were used to establish the content validity: (1) Content Validity IndexItem (I-CVIs) $>0.78$, and (2) Content Validity Index-Scale $(\mathrm{S}-\mathrm{CVI})>0.90$. The results of the I-CVI confirmed that $83 \%$ $(n=25)$ of the items were scored as acceptable (values ranged from 0.83 to 1.00 ). I-CVIs of 5 items of the 30 items did not meet the cut-off of 0.78 . These five items were removed from the tool, resulting in a 25 -items version. The content validity of the overall tool was established based on the S-CVI score which was 0.92 .

\section{Creation of a pilot version of the questionnaire}

A group of 20 caregivers, whose characteristics matching the inclusion criteria, participated in the pilot testing to evaluate the comprehensibility and feasibility during administration. The time needed to complete the tool was 10-15 min. No further changes in structure or content were in order. Subsequently, the pilot draft of the tool was composted of 25 items written in Arabic covering the seven domains, as follows: Psychological Well-being - PW (5 items); Self-Stigma - SS (7 items); Relationships with Family and Relatives - RFR (3 items); Relationships with Psychiatric Health Team - RPHT (3 items); Physical Health - PH (2 items); Psychological Burden - PB (2 items) and Financial Burden - FB (3 items). A Likert-type format was used with 5-points, ranging from 1 (strongly disagree) to 5 (strongly agree).

Construct validity, reliability and some aspects of external validity were tested for QLSSoSPC tool.

Ceiling and floor effects were considered to evaluate the skewness of the answer distribution. The floor effect reflects the proportion of participants who have the worst possible total score for the tool, as well as the ceiling effect reflects the proportion with the best score that is possible for the tool. A high ceiling or floor effect limits the result of a tool for the reason that persons falling within the highest and lowest averages cannot obtain better or worse scores. ${ }^{28}$ Accordingly, it results in an absolute lack of tool sensitivity.

\section{Data analysis}

Statistical Package for Social Sciences (SPSS) software (Version 21.0) and WINSTEPS software 3.72 .3 based on the Rasch model were used to assess the tool items' appropriateness and suitability. ${ }^{29}$

\section{Ethical considerations}

The current study was approved by the Research Ethics Committee of King Khalid University with approval number: (ECM\#2019-57)-( HAPO-06-B-001). 


\section{Results}

\section{Socio-demographic and clinical characteristics}

A total of 205 family caregivers used the self-administered tools with a response rate of $81 \%$. The sample mean age was 55 years with a standard deviation of 9.4 years and ranged between 30 and 65 years. A total of 33\% were male caregivers, whereas $67 \%$ were female caregivers. Nearly half of the study participants had a job $(58.5 \%, n=120)$. The majority of the sample had more than three members in their family $(85.9 \%, n=176)$. For patients with schizophrenia, the mean of age since diagnosis was 22.3 years with a standard deviation of 6.7 years (see Table 1 ).

\section{Scoring}

For each caregiver, a score for each tool domain was obtained by calculating the mean of the tool item scores on the domain. But if less than half of the QLSSoSPC items were missing, non-missing items mean was given for the missing items. The scores of all domains were linearly standardised to a $0-100$ scale, with 100 score indicating the highest level of quality of life and 0 the lowest.

TABLE 1: Caregiver's socio-demographic characteristics and clinical information $(n=205)$.

\begin{tabular}{lcc}
\hline Variables & $\boldsymbol{N}$ & $\%$ \\
\hline The caregivers & 55 & \\
Mean of age (years) \pm s.d. & 68 & 9.4 \\
Gender (Male) & 176 & 33.2 \\
Caregivers' family members $\geq 3$ & 120 & 85.9 \\
Have Occupation & & 58.5 \\
The patients with schizophrenia & 8.7 & \\
Schizophrenia duration (years) \pm s.d. & 22.3 & 9.6 \\
Age (mean) at diagnosis (years) \pm s.d. & & 6.7 \\
Types of schizophrenia & 27 & \\
Undifferentiated & 9 & 13 \\
Disorganised & 40 & 4.3 \\
Residual & 10 & 19.4 \\
Schizoaffective & 119 & 5.1 \\
Paranoid & & 58.2 \\
\hline
\end{tabular}

s.d., standard deviation.

\section{Construct validity}

Item iteration process confirmed the hypothesised sevendimension factor structure with 25-items. The scalability was considered acceptable and all items showed a good fit to the Rasch model within each dimension, with no items showing an item goodness-of-fit statistics (INFIT) outside of the acceptable range (value between 0.7 and 1.2). Also, all items on each factor were from the same domain, and the total explained variance by all factors together was $75.6 \%$. For each domain, name was given according to its items, as the followings: PW (5 items); SS (7 items); RFR (3 items); RPHT (3 items); PH(2 items); PB (2 items) and FB (3 items) (see Appendix 1). The results established an absence of DIF related to participants' age and gender which supported the invariance of the tool item calibrations.

\section{Reliability}

The reliability was determined using internal consistency methods. The internal consistency for the whole sample and each subscale was determined by Cronbach's $\alpha$. Cronbach's $\alpha$ of about 0.7 is considered sufficient. Furthermore, the following criteria were applied to recognise poorly functioning items: (1) a correlation of less than 0.30 between an item and the subscale score or (b) an increase of $>0.10$ in the total questionnaire reliability when the item was deleted. $^{30}$

Cronbach's $\alpha$ ranged from 0.80 and 0.91 in the whole sample. All the corrected item-total correlations were found to be $>0.30$, indicating high internal consistency. The deletion of any of the 25 items did not increase the internal consistency of any of the seven dimensions. Item internal consistency was satisfactory for all dimensions ranging from 0.63 to 0.94 for each item. The correlation of each item with its associated dimension was higher than its correlation with the other dimensions (item discriminant validity) (See Table 2).

TABLE 2: Characteristics of tool domains $(n=205)$.

\begin{tabular}{|c|c|c|c|c|c|c|c|c|}
\hline Domain & PW & SS & RFR & RPHT & $\mathrm{PH}$ & PB & FB & Index \\
\hline Mean & 52.8 & 61.7 & 68.4 & 56.8 & 61.8 & 68.1 & 61.1 & - \\
\hline s.d. & 17.4 & 15.3 & 20.8 & 21.7 & 19.6 & 21.3 & 10.3 & - \\
\hline \multicolumn{9}{|c|}{ Item-internal consistency } \\
\hline Min-Max & $0.82-0.91$ & $0.63-0.77$ & $0.73-0.90$ & $0.91-0.94$ & $0.92-0.93$ & $0.92-0.93$ & $0.79-0.87$ & $\mathrm{~N} / \mathrm{A}$ \\
\hline \multicolumn{9}{|c|}{ Item discriminant validity } \\
\hline Min-Max & $0.01-0.57$ & $0.00-0.50$ & $0.00-0.38$ & $0.00-0.28$ & $0.00-0.40$ & $0.05-0.33$ & $0.06-0.41$ & N/A \\
\hline \multicolumn{9}{|l|}{ Missing values } \\
\hline$\% \mathrm{MV}$ & 0.3 & 0.3 & 7.6 & 5.2 & 4.6 & 4.8 & 5.2 & 18.9 \\
\hline \multicolumn{9}{|l|}{ Floor effect } \\
\hline$\%$ & 10.6 & 12.1 & 7.6 & 7.1 & 16.5 & 7.7 & 10.1 & 10.3 \\
\hline \multicolumn{9}{|l|}{ Ceiling effect } \\
\hline$\%$ & 6.0 & 13.8 & 44.3 & 10.8 & 9.7 & 10.1 & 32.6 & 15.4 \\
\hline Cronbach's alpha & 0.91 & 0.87 & 0.81 & 0.91 & 0.86 & 0.87 & 0.80 & 0.86 \\
\hline \multicolumn{9}{|c|}{ Rasch statistics (INFIT) } \\
\hline
\end{tabular}

PW, psychological well-being; SS, self-stigma; RFR, relationships with family and relatives; RPHT, relationships with psychiatric health team; PH, physical health; PB, psychological burden; FB, financial burden; N/A, not applicable; INFIT: item goodness-of-fit statistics, \% MV: missing values, s.d., standard deviation. 


\section{External validity}

The QLSSoSPC index was significantly correlated with all WHOQOL-BREF domains scores ( $\mathrm{rs}=0.18-0.43)$. However, only two domains of the QLSSoSPC (PW, SS) showed medium to high correlations with domain scores of the WHOQOL-BREF assessing PW and SS: PW-PH, PW-Ps, PW-SR, PW-E and SS-Ps. On the contrary, the specific domains of the QLSSoSPC: PH, PB and FB were uncorrelated or weakly correlated with the domains of WHOQOL-BREF (See Table 3).

In comparison between male and female participants, results showed significant differences for the following two domains: PW for male was 58.2 (18.3) and female 50.3 (16.5), $p=0.003$; and RFR for male was 76.6 (16.0) and female 64.2 (24.0), $p<0.0001$. Family consists of three and more members, who were associated with a higher tool score for FB and the result of index. Caregivers without job presented a significant high level for RFR as well as a lower level of PB. Moreover, caregivers of patients with paranoid schizophrenia type showed lower tool scores (RPHT and the index). And the highest total scores for RPHT and the index are shown in Table 4.

The SS, RFR and PB domains scores significantly correlated with the study caregiver's age $0.23,0.13$ and -0.24 , respectively. The patient age at diagnosis of schizophrenia had also significant correlation $\mathrm{rs}=(0.13-0.23)$ with SS, RFR, PH, FB and the index (See Table 5).

\section{Discussion}

The main aims of this study were to develop and validate a tool designed to assess the QLSSoSPC. Developing and validating a new tool to assess multiple important domains related to QLSSoSPC arose from the fact that most of the available tools were not addressing self-stigma as an influencing factor. ${ }^{31}$ Thus, providing a reliable and valid tool for the schizophrenia caregivers is needed. The QLSSoSPC assesses significant factors that must be discussed when planning care for patients with schizophrenia. Neong and Rashid $^{31}$ recommended that the healthcare givers and policymakers recognise the factors that affect the caregivers quality of life and SS, bearing in mind that more patients with psychiatric health disorders are being cared for in the patients' community.

TABLE 3: Spearman's correlations between quality of life and self-stigma of schizophrenia patient's caregiver and WHOQOL-BREF scores $(n=205)$.

\begin{tabular}{lcccccccc}
\hline Domain & PW & SS & RFR & RPHT & PH & PB & FB & Index \\
\hline PH & $0.45^{* *}$ & $0.26^{* *}$ & $0.13^{*}$ & 0.10 & $0.17^{* *}$ & 0.12 & $0.14^{*}$ & $0.36^{* *}$ \\
PS & $0.52^{* *}$ & $0.40^{* *}$ & $0.12^{*}$ & 0.10 & $0.22^{* *}$ & 0.10 & 0.06 & $0.36^{* *}$ \\
SR & $0.42^{* *}$ & $0.27 * *$ & $0.14^{*}$ & 0.05 & $0.17^{* *}$ & $0.13^{*}$ & $0.12^{*}$ & $0.32^{* *}$ \\
E & $0.36^{* *}$ & $0.24 * *$ & 0.15 & -0.06 & 0.07 & 0.05 & -0.00 & $0.16^{*}$ \\
\hline
\end{tabular}

WHOQOL-BREF, the Arabic version of the Short-form of the World Health Organization's quality of life; PW, psychological well-being; SS, self-stigma; RFR, relationships with family and relatives; RPHT, relationships with psychiatric health team; PH, physical health; PB, relationships; $E$, environment.

$*, p<0.05 ; * *, p<0.01$.

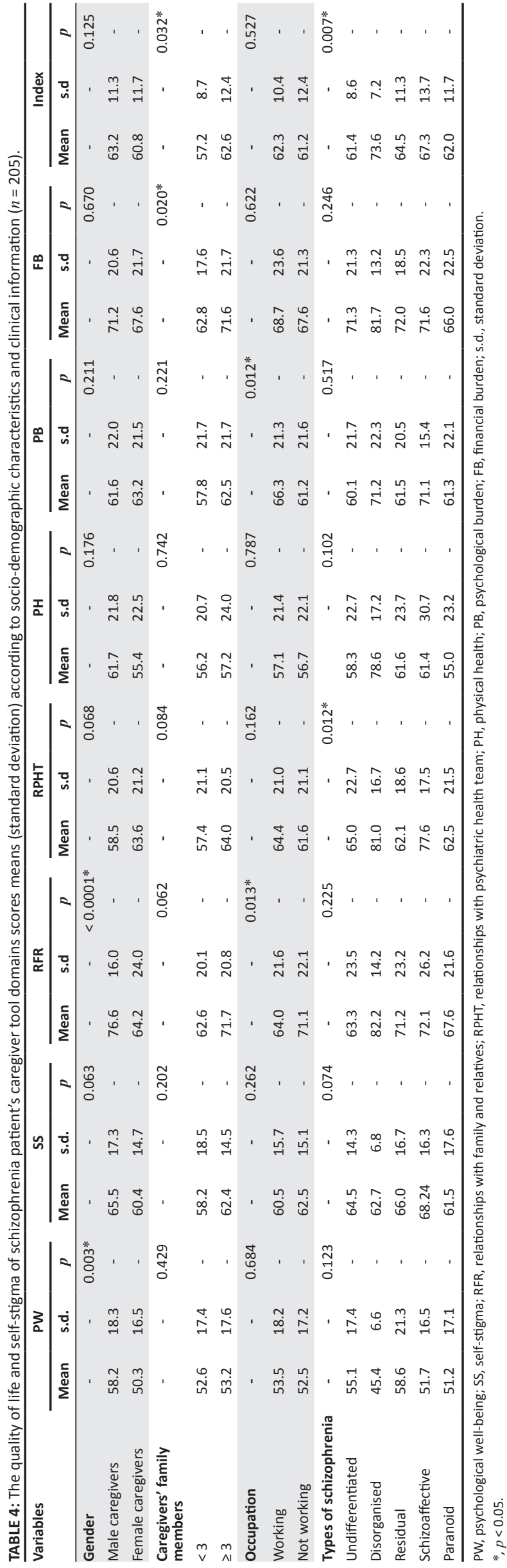

Open Access 
TABLE 5: Spearman correlation of the total score of the quality of life and self-stigma of the schizophrenia patient's caregiver tool with caregiver's age and patient's age at diagnosis.

\begin{tabular}{lcccccccc}
\hline Variables & PW & SS & RFR & RPHT & PH & PB & FB & Index \\
\hline Caregiver's age & 0.11 & $0.23^{* *}$ & $0.13^{*}$ & -0.02 & -0.06 & $-0.24 * *$ & 0.08 & 0.02 \\
$\begin{array}{l}\text { Patient age at } \\
\text { diagnosis }\end{array}$ & 0.06 & $0.17^{*}$ & $0.13^{*}$ & 0.05 & $0.17^{* *}$ & 0.07 & $0.16^{*}$ & $0.23^{* *}$ \\
\hline
\end{tabular}

PW, psychological well-being; SS, self-stigma; RFR, relationships with family and relatives; RPHT, relationships with psychiatric health team; PH, physical health; PB, psychological burden; $\mathrm{FB}$, financial burden.

$*, p<0.05$ and $* *, p<0.01$

Furthermore, SS is considered as the psychological health issue that can be experienced by mentally ill patient's caregivers, and it is found that caregivers' SS negatively affects patients' adherence, as well as treatment seeking and rehabilitation. ${ }^{32}$ Mak and Cheung ${ }^{33}$ studied the relationship between the SS and quality of life amongst mental disorders patients' caregivers, who found that SS was correlated to caregivers' quality of life. The current available tools for schizophrenia caregivers are essentially based only on the experts' or clinicians' viewpoint, except for the tools developed by Szmukler, Burgess ${ }^{34}$ and Richieri, Boyer. ${ }^{35}$ The components of the QLSSoSPC integrate factors of great significance to patients and are considerably different from other quality of life tools.

Correspondingly, some factors of the QLSSoSPC are similar to those referred to in the literature and the existing tools, such as PW, RFR, RPHT, PH, PB and FB. However, some dimensions, like SS, emerged as a unique concern for the schizophrenia caregivers. The study's findings indicate that the QLSSoSPC tool is a reliable and valid tool for assessing the caregivers' quality of life and SS. All the subscales of the QLSSoSPC were internally consistent and mutually exclusive. The Cronbach's $\alpha$-coefficients for all items ranged from 0.80 to 0.9 , indicating excellent levels of internal consistency.

The findings obtained from Rasch analysis identified seven domains for the QLSSoSPC tool. The majority of the items loaded on their relevant domain, thus confirming the construct validity of the QLSSoSPC tool. Furthermore, the findings obtained indicated that the 25-items of the QLSSoSPC tool fit the data significantly and support the proposed structure. Thus, strong statistical evidence was available to support the construct validity of the seven domains. The psychometric properties for the QLSSoSPC were similar to a few scales developed to assess various sets of factors related to quality of life for schizophrenia caregivers. $^{34,35}$ The development procedure used in the current study was guided by recommendations made by Morgado and Meireles. ${ }^{27}$

An interesting finding from this study was the positive relationship between caregiver's quality of life and the caregiver's age for SS and PB domains. Zhai and $\mathrm{Du}^{36}$ demonstrated similar results that SS is significantly higher for age 35 years or younger. Moreover, it is found that younger participants' age was significantly associated with depressive symptoms. ${ }^{37}$ We found that male caregivers had better quality of life than female caregivers, particularly for the domains of PW and RFR. However, the majority of the studies found that female caregivers had lower quality of life. ${ }^{5,7}$

We also found that family caregiver's quality of life was higher for caregivers who have more than three family individuals; this could be attributed to the help and support for caregivers by other family members. This finding converges with $\mathrm{Li}$, Lambert ${ }^{8}$ study in which they found a positive correlation between caregivers' quality of life and patient's family members' numbers. The findings of the current study indicated significant associations between $\mathrm{PB}$ and family and relatives domains. This matches with the findings from a cross-sectional study conducted in Indonesia by Boyer and Caqueo-Urízar ${ }^{5}$ who found that employment condition and social support positively affected quality of life.

One more finding was related to patient's age at diagnosis of schizophrenia. In our study, the higher quality of life of the caregivers was associated with older age of patient diagnosis. Similarly, Li, Lambert ${ }^{8}$ found that patient diagnosis was significantly associated with older age. The suitability of QLSSoSPC is good. There was low level of missing data that were between $0.3 \%$ and $7.6 \%$ for all domains. The expected average time to use the tool is likely to be nearly $5 \mathrm{~min}$ that will enhance its use in psychiatric research and clinical practice.

There were several limitations to the current study. Firstly, the stability of the tool was not measured over time. Secondly, cross-validation studies are required to confirm the factor structure of the QLSSoSPC tool, and to establish the reliability and validity in other populations. Thirdly, the sample may not be representative because of the self-selection of the conveniently selected participants.

\section{Conclusion}

The QLSSoSPC tool is a novel tool that combined in its structure the factor related to SS that was not measured before by any tool. The existing study tool measures quality of life and SS for schizophrenia caregivers. Also, it is not proposed to substitute the available tools. Rather, the QLSSoSPC tool adds remarkable information to psychiatric healthcare and enables researchers to assess the schizophrenia patients' caregivers' quality of life and SS by using one tool. It would be crucial to investigate the reproducibility of the existing study results. However, the QLSSoSPC tool psychometric properties are supported by the rigor of the statistical methods used in the existing study. The originality of the QLSSoSPC tool may be valuable in psychiatric practice and research scope.

\section{Acknowledgements}

The authors would like to express their gratitude to King Khalid University, Saudi Arabia, for providing administrative and technical support. 


\section{Competing interests}

The authors declare that they have no financial or personal relationships that may have inappropriately influenced them in writing this article.

\section{Authors' contributions}

Both Z.A. and M.A. contributed to the study design, analysis and interpretation of data, drafted the article, and read and approved the final article.

\section{Funding information}

This research received no specific grant from any funding agency in the public, commercial or not-for-profit sectors.

\section{Data availability}

The data that support the findings of this study are available from the corresponding author, Z.A., upon reasonable request.

\section{Disclaimer}

The views expressed in this article are those of the authors and not an official position of the institution where the study was carried out.

\section{References}

1. McGrath J, Saha S, Chant D, Welham J. Schizophrenia: A concise overview of incidence, prevalence, and mortality. Epidemiol Rev. 2008;30(1):67-76. https:// doi.org/10.1093/epirev/mxn001

2. Leung DA, Chue P. Sex differences in schizophrenia, a review of the literature. Acta Psychiatr Scand. 2000;101(401):3-38. https://doi.org/10.1111/j.0065-1591. 2000.0ap25.x

3. Brady N, McCain GC. Living with schizophrenia: A family perspective. Online J Issues Nurs. 2004;10(1).

4. Kaushik P, Bhatia M. Burden and quality of life in spouses of patients with schizophrenia and bipolar disorder. Delhi Psychiatr J. 2013;16(1):83-89.

5. Boyer L, Caqueo-Urízar A, Richieri R, et al. Quality of life among caregivers of patients with schizophrenia: A cross-cultural comparison of Chilean and French families. BMC Fam Pract. 2012;13(1):42. https://doi.org/10.1186/1471-2296-13-42

6. Awad AG, Voruganti LN. The burden of schizophrenia on caregivers Pharmacoeconomics. 2008;26(2):149-162. https://doi.org/10.2165/00019053200826020-00005

7. Caqueo-Urízar A, Gutiérrez-Maldonado J, Miranda-Castillo C. Quality of life in caregivers of patients with schizophrenia: A literature review. Health Qual Life Outcome. 2009;7(1):84. https://doi.org/10.1186/1477-7525-7-84

8. Li J, Lambert CE, Lambert VA. Predictors of family caregivers' burden and quality of life when providing care for a family member with schizophrenia in the People's Republic of China. Nurs Health Sci. 2007;9(3):192-198. https://doi.org/10.1111/ j.1442-2018.2007.00327.x

9. Reine G, Lancon C, Simeoni M, Duplan S, Auquier P. Caregiver burden in relatives of persons with schizophrenia: An overview of measure instruments. L'encéphale. 2003;29(2):137.

10. Velligan $D$, Weiden $P$, Sajatovic $M$, et al. Expert consensus panel on adherence problems in serious and persistent mental illness. The expert consensus guideline
series: Adherence problems in patients with serious and persistent mental illness. $\mathrm{J}$ Clin Psychiatry. 2009;70(suppl 4):1-46. https://doi.org/10.4088/JCP.7090su1cj

11. Ohaeri JU. Caregiver burden and psychotic patients' perception of social support in a Nigerian setting. Social Psychiatr Psychiatr Epidemiol. 2001;36(2):86-93. https://doi.org/10.1007/s001270050294

12. Perlick DA, Rosenheck RA, Kaczynski R, Swartz MS, Cañive JM, Lieberman JA Special section on CATIE baseline data: Components and correlates of family burden in schizophrenia. Psychiatr Serv. 2006;57(8):1117-1125. https://doi. org/10.1176/ps.2006.57.8.1117
13. Becker S, Al Zaid K, Al Faris E. Screening for somatization and depression in Saudi Arabia: A validation study of the $\mathrm{PHQ}$ in primary care. Int I Psychiatr Med. 2002;32(3):271-283. https://doi.org/10.2190/XTDD-8L18-P9E0-JYRV

14. Larson JE, Corrigan P. The stigma of families with mental illness. Academic Psychiatr. 2008;32(2):87-91. https://doi.org/10.1176/appi.ap.32.2.87

15. Schene AH, Van Wijngaarden B, Koeter MW. Family caregiving in schizophrenia: Domains and distress. Schizophr Bull. 1998;24(4):609-618. https://doi. org/10.1093/oxfordjournals.schbul.a033352

16. Tessler RC, Gamache G. Toolkit for evaluating family experiences with severe mental illness. Cambridge: Human Services Resources Institutes; 1995.

17. Kadri N, Manoudi F, Berrada S, Moussaoui D. Stigma impact on Moroccan families of patients with schizophrenia. Can J Psychiatr. 2004;49(9):625-629. https://doi. org/10.1177/070674370404900909

18. Mak WW, Cheung RY. Psychological distress and subjective burden of caregivers of people with mental illness: The role of affiliate stigma and face concern Community Ment Health J. 2012;48(3):270-274. https://doi.org/10.1007/ s10597-011-9422-9

19. Werner P, Mittelman MS, Goldstein D, Heinik J. Family stigma and caregiver burden in Alzheimer's disease. Gerontologist. 2012;52(1):89-97. https://doi. org/10.1093/geront/gnr117

20. Zhang $Y$, Subramaniam M, Lee SP, et al. Affiliate stigma and its association with quality of life among caregivers of relatives with mental illness in Singapore. Psychiatr Res. 2018;265:55-61. https://doi.org/10.1016/j.psychres.2018.04.044

21. Picco L, Pang S, Lau YW, et al. Internalized stigma among psychiatric outpatients: Associations with quality of life, functioning, hope and self-esteem. PsychiatrRes.2016;246:500-506. https://doi.org/10.1016/j.psychres.2016.10.041

22. Basheer S, Anurag K, Garg R, Kumar R, Vashisht S. Quality of life of caregivers of mentally ill patients in a tertiary care hospital. Ind Psychiatr J. 2015;24(2):144 https://doi.org/10.4103/0972-6748.181721

23. Polit DF, Beck CT. Essentials of nursing research: Appraising evidence for nursing practice. Philadelphia: Lippincott Williams \& Wilkins; 2009.

24. Nunnally JC. An overview of psychological measurement. In: Benjamin BW, editor Clinical diagnosis of mental disorders. New York: Springer, 1978; p. 97-146.

25. Whoqol Group. Development of the World Health Organization WHOQOL-BREF quality of life assessment. Psychol Med. 1998 May;28(3):551-558.

26. Cappelleri JC, Lundy JJ, Hays RD. Overview of classical test theory and item response theory for the quantitative assessment of items in developing patientreported outcomes measures. Clin Therapeut. 2014;36(5):648-662. https://doi. org/10.1016/j.clinthera.2014.04.006

27. Morgado FF, Meireles JF, Neves CM, Amaral AC, Ferreira ME. Scale development: Ten main limitations and recommendations to improve future research practices. Psicologia: Reflexão e Crítica. 2018;30(1):3. https://doi.org/10.1186/s41155-017 0059-7

28. Rhemtulla M, Brosseau-Liard PÉ, Savalei V. When can categorical variables be treated as continuous? A comparison of robust continuous and categorical SEM estimation methods under suboptimal conditions. Psychol Meth. 2012;17(3):354 https://doi.org/10.1037/a0029315

29. Linacre J. Realiability and separations. A users guide to Winsteps/Ministep Rasch-Model Computer Programs. Chicago, IL: Winsteps.com; 2007.

30. Boateng GO, Neilands TB, Frongillo EA, Melgar-Quiñonez HR, Young SL. Best practices for developing and validating scales for health, social, and behavioral research: A primer. Frontiers in Public Health. 2018 Jun 11;6:149.

31. Neong S, Rashid A. Quality of life of caregivers of patients with psychiatric illness in Penang. J Clin Psychiatry Cog Psychol 2018;2(1):12. https://doi.org/10.35841/ clinical-psychiaetry.2.1.12-18

32. Girma E, Möller-Leimkühler AM, Dehning S, Mueller N, Tesfaye M, Froeschl G. Self-stigma among caregivers of people with mental illness: Toward caregivers' empowerment. J Multidiscip Healthc. 2014;7:37. https://doi.org/10.2147/JMDH. S57259

33. Mak WW, Cheung RY. Affiliate stigma among caregivers of people with intellectual disability or mental illness. J Appl Res Intellect Disabil. 2008;21(6):532-545. https://doi.org/10.1111/j.1468-3148.2008.00426.x

34. Szmukler G, Burgess P, Herrman H, Bloch S, Benson A, Colusa S. Caring for relatives with serious mental illness: The development of the experience of caregiving inventory. Soc Psychiatr Psychiatr Epidemiol. 1996;31(3-4):137-148. https://doi. org/10.1007/BF00785760

35. Richieri R, Boyer L, Reine G, et al. The Schizophrenia Caregiver Quality of Life questionnaire (S-CGQoL): Development and validation of an instrument to measure quality of life of caregivers of individuals with schizophrenia. Schizophr Res. 2011;126(1-3):192-201. https://doi.org/10.1016/j.schres. 2010.08.037

36. Zhai Y, Du X. Mental health care for international Chinese students affected by the COVID-19 outbreak. Lancet Psychiatr. 2020;7(4):e22. https://doi.org/10.1016/ S2215-0366(20)30089-4

37. Magaña SM, Ramirez Garcia Jl, Hernández MG, Cortez R. Psychological distress among Latino family caregivers of adults with schizophrenia: The roles of burden and stigma. Psychiatr Serv. 2007;58(3):378-384. https://doi.org/10.1176/ ps.2007.58.3.378 


\section{Appendix 1}

TABLE 1-A1: The Arabic and English version of QLSSoSPC tool.

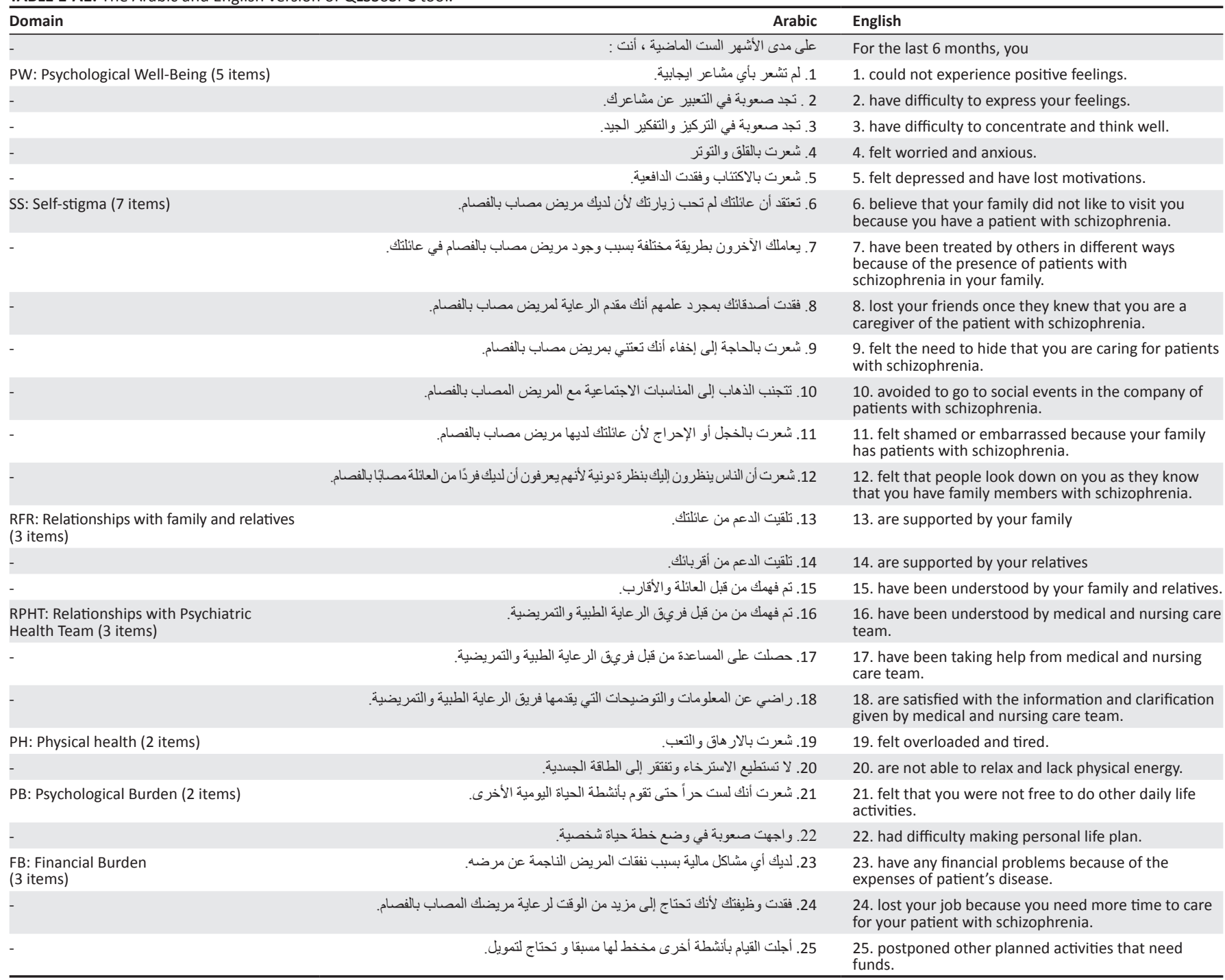

